\title{
An Area Localization Algorithm Using RFID
}

\author{
Yaoqi Du, Zhiying Yang* \\ Department of Computer Science \& Technology, Shanghai Maritime University, Shanghai, China
}

Email address:

du_yaoqi@hotmail.com (Yaoqi Du),zyyang@shmtu.edu.cn (Zhiying Yang)

To cite this article:

Yaoqi Du, Zhiying Yang. An Area Localization Algorithm Using RFID. Internet of Things and Cloud Computing. Vol. 3, No. 4, 2015, pp. 82-87. doi: 10.11648/j.iotcc.20150304.11

\begin{abstract}
There is a growing interest in the research of localization system based on radio frequency identification (RFID) technology. In some application scenarios, such as smart shelves, tend to have the demand for the exact location of the target object like books. Existing RFID localization algorithm usually assumes that the application environment is indoor, which couldn't locate the target in a small enough space. Different from the localization system which gives the specific coordinates of the tracking tag like LANDMARC, this paper present an area localization algorithm, ALLGET. Based on grouping of reference tags, ALLGET locate the tracking tag into a small area. Our experimental results show that ALLGET can get a better accuracy than LANDMARC in the same condition.
\end{abstract}

Keywords: RFID, Area Localization, ALLGET, LANDMARC

\section{Introduction}

The rapid proliferation of RFID technology drives a series of application. Localization system is one of them. In many cases we need to know the position of objects. Under these circumstances, there is a growing interest in the research of localization system based on RFID technology. In the past few years, many systems and algorithm have been designed for automatic location-sensing using RFID or similar techniques. Triangulation and scene analysis are the two of principle techniques to approach that $[9,10]$. With the method of multi-point collaboration, Triangulation locate objects through distance, angle and other elements. TOA/TDOA and AOA are representative algorithms using triangulation. Scene analysis method uses environmental information to help to locate. The most well-known location-sensing system using scene analysis is LANDMARC [1], which is designed by the research team of The Hong Kong University of Science and Technology in 2004. Indoor positioning system based on LANDMARC algorithm introduces reference tag, and uses these extra fixed location reference tags to help to locate. With these reference tags as reference point, the main idea of LANDMARC is using k-Nearest Neighbor to improve the accuracy of the system.

The result of LANDMARC is the estimated coordinates of tracking tags. But in practical indoor application, coordinates are usually not practical. In many cases we just need to know in which part of the area the target is located. For example, on which one does a book put among many shelves. In certain application scenarios like this, we have to analysis the coordinates in order to get the information we need. But in fact, due to the placement of reference tags is known, the selection of $\mathrm{k}$ reference tags in LANDMARC already contains certain area information. In order to make full use of the area information contained in the reference tag, we proposed an area localization algorithm based on grouping of reference tags, ALLGET. With grouped reference tags corresponded to the actual area division, the ALLGET system can directly locates the tracking tags in a small area without coordinates.

At present, there are several kinds of location-sensing system, such as Cricket, RADAR and LANDMARC. Section 2 will give an overview of these technologies and some related work. Section 3 introduces a prototype ALLGET system, and explains how it works. Section 4 presents the simulation results of the prototype ALLGET system under different conditions, and compared with the LANDMARC system under the same condition. Based on the simulation, we analysis the main reason that impact on the system performance. In section 5, we conclude the paper and describe some future research.

\section{Related Work}

At present, there are several location-sensing system using wireless technology, such as The Cricket Location Support 
System developed by MIT Oxygen project, RADAR by Microsoft, SpotON developed at the University of Washington, LANDMARC system developed at The Hong Kong University of science and technology, etc. Each of these technologies have their own strengths as well as limitations.

Among these systems, Cricket system is a scheme based on the references node of grid layout, which uses TDOA algorithm to calculate distance [5]. It is the earliest loosely coupled system of the MIT Oxygen development project. A known reference node (called anchor nodes) and a target node's position consist of system. Anchor nodes transmit radio frequency signal and ultrasonic signal continuously. Radio frequency signal contains the anchor nodes' location information and its ID. TDOA algorithm used to calculate the distance between the target node and its anchor node. When the target node obtains three or more anchors' signals, it can use trilateration method to calculate the location of the target node.

RADAR positioning system is a system that estimates distance based on the wireless signal strength [4], followed by estimating the destination location by using multiple distance values. RADAR system is a tightly coupled positioning system. The system requires databases that are built from every radio frequency signal in the environment. System runs by applying nodes to collect data in processing centre, followed by comparing the results with the contents from the database.

SpotON system is based on clustering algorithm's received signal strength for three-dimensional position sensor [6]. According to small cluster, this system is able to obtain higher accuracy than the triangulation algorithm of positioning performance. But this system's positioning accuracy is still not high enough, and the system itself is inadequate.

LANDMARC system introduces reference tag on a known location [1], which aims to correct the pending uncertainties in the positioning tag. With these fixed location reference tags, the main idea of LANDMARC is using k-Nearest Neighbor to improve the accuracy of the system. LANDMARC system introduces an $\mathrm{E}$ value as the Euclidian distance in signal strength between a tracking tag and a reference tag. And the nearer reference tag to the tracking tag is supposed to have a smaller E value. Several nearest neighbors are chosen depend on the E values. This system not only improves the positioning accuracy with fewer reader devices, but also reduces the cost of system.

\section{Allget Approach}

ALLGET takes use of reference tag to assist position. Meanwhile, ALLGET divides the reference tags into groups, making a correspondent relationship between actual zone and reference tags in order to make fully use of location information provided by the reference tags and reduce errors caused by transformed information.

\subsection{System Setup}

We build a prototype system based on a bookshelf. Suppose that the bookshelf has a total of six layers, each layer divided into four frames. We place reference tags on each vertices of a frame. For each frame, there are eight reference tags placed around it. There are 24 frames in the bookshelf, using 70 reference tags in total. 4 RFID readers are placed on four vertices of a side of the bookshelf. Figure 1 is a schematic diagram of the prototype system.

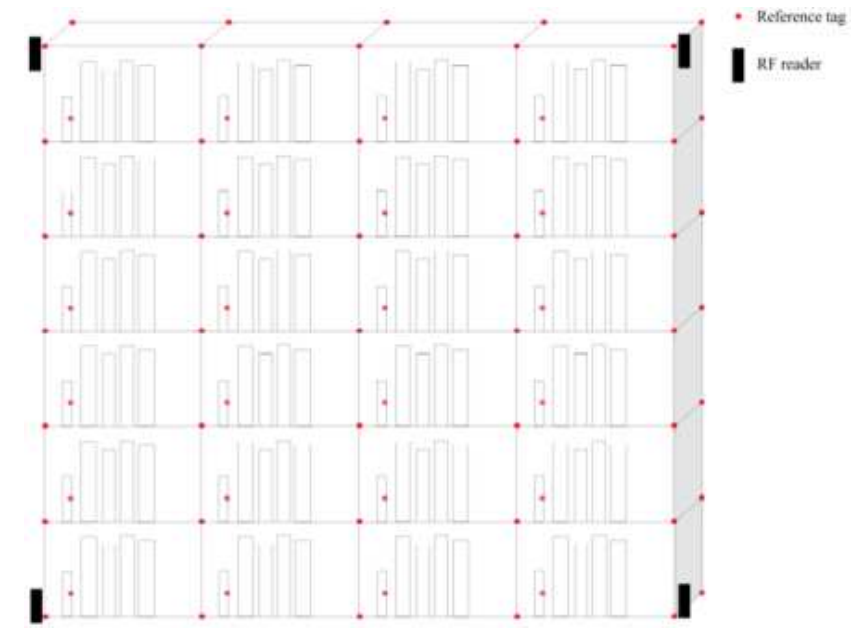

Figure 1. Placement of $R F$ readers and tags in prototype system.

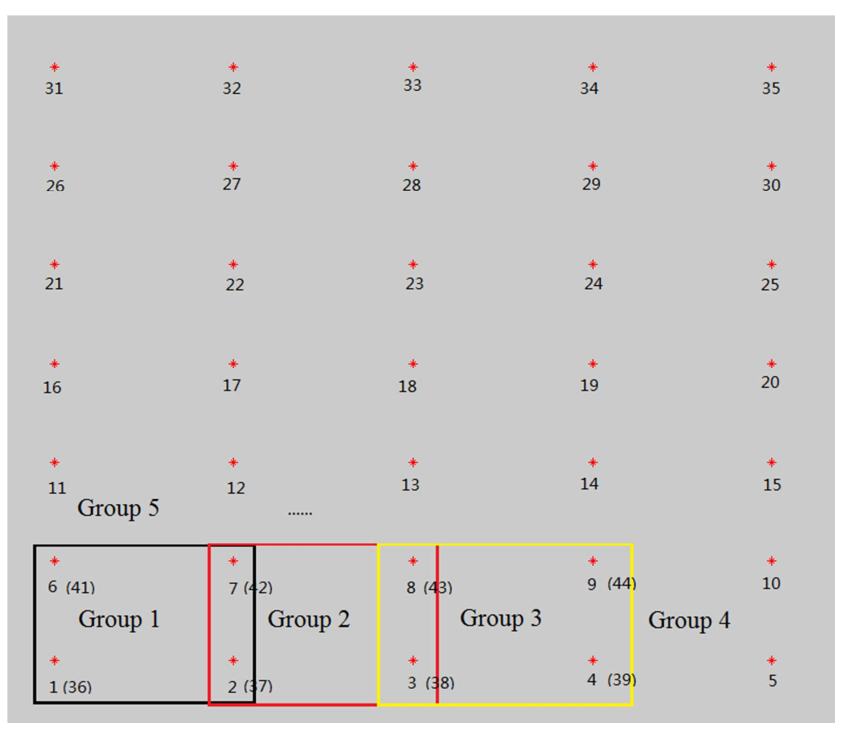

Figure 2. Grouping of reference tags (front view).

\subsection{Methodology}

ALLGET uses RFID reader device to read the signal strength value of tags, which carries out a positioning analysis. The system requires ultra high frequency RFID tags in order to achieve enough reading distances. Signal strength value does not directly reflect the positional relationship between the tags and the reader device. In order to determine the target's position, a plan for the signal strengths between the tags need to be established to determine the position by comparison.

Suppose there are $\mathrm{m}$ RF readers, $\mathrm{n}$ reference tags and $\mathrm{u}$ tracking tags. According to LANDMARC, we can use

$$
E_{j}=\sqrt{\sum_{i=1}^{m}\left(\theta_{i}-S_{i}\right)^{2}}, \mathrm{i} \in(1, \mathrm{~m}), \mathrm{j} \in(1, \mathrm{n})
$$


where $\theta_{i}$ denotes the signal strength of the reference tag perceived on reader i and $S_{i}$ denotes the signal strength of the tracking tag perceived on reader $i$, as the Euclidian distance in signal strength between a tracking tag and a reference tag [1].

In order to reduce the error impact that the tag signal strength has, the system uses a number of reader devices to cover the entire area that people wish to locate. This ensures that each target tag and reference tag can be read with multiple reader devices. In this way, it can reduce positioning errors because $\mathrm{E}$ value given by couples of reader devices is much

$$
\left\{\begin{array}{c}
j_{1}=t+t \backslash 5, j_{2}=t+1+t \backslash 5, j_{3}=t+5+t \backslash 5, j_{4}=t+6+t \backslash 5 \\
j_{5}=t+35+t \backslash 5, j_{6}=t+36+t \backslash 5, j_{7}=t+40+t \backslash 5, j_{8}=t+41+t \backslash 5
\end{array}\right.
$$

In this formula, $j_{n}$ is the number of $t^{\text {th }}$ reference tag in group t. This formula is only suitable for the original of the system. When the placement of reference tags changes, the relationship will change, too.

Note that reference tags are repeated between adjacent groups. Each set of reference tag that corresponds to the actual area is called a grid. Assume that reference tags have been divided into $\mathrm{T}$ groups.

Reference tag group sets up a correspondent relationship between the tag and the actual area. For one positioning tag that relies on an entire area, we only need to work out which group that the tag belongs to in order to locate it within a smaller area. Since the reference tag has smaller E value for the closer positioning tag, we can compare the distance between different groups and the positioning tag. The group has shortest distance represents the position result that the positioning tag owns.

In order to be able to have group comparisons, reference volumes are needed to satisfy the following requirements: it must be a monotone function of $\mathrm{E}$, which could eliminate the negative impact due to the error from $E$ to a certain extent and avoid enlarger error under grouping situation. By comparison, we choose the second-order weights to replace E value. Define the corresponding metric

$$
w_{j}=\frac{\frac{1}{E_{i}^{2}}}{\sum_{i=1}^{n} \frac{1}{E_{i}^{2}}}, \mathrm{j} \in(1, \mathrm{n})
$$

as the $\mathrm{j}^{\text {th }}$ reference tag's positioning tag. The metric here is different from the same metric in LANDMARC algorithm [1], which is calculated for all reference tags as a whole rather than $\mathrm{k}$ neighbor tag(s). In this case, $\mathrm{n}$ is equal to all reference tags' numbers in the system. The purpose of inserted metric is to reduce the error impact due to signal strength during comparison. Whereas, the LANDMARC metric is to ensure the proportion of different nearest neighbor tags' location takes in the final result. It could be concluded that inserted metric is fundamentally different from the LANDMARC metric.

After calculating metrics for each reference's corresponding tag, the metrics are divided into groups. Define the sum of metric

$$
W G_{t}=\sum_{i=1}^{s} W_{i}, \mathrm{t} \in(1, \mathrm{~T})
$$

closer to the real situation.

Since the method of grouping is related with the scenario's practical application, the case shown below is based on the original of the system. As shown in figure 2, number $1 / 2 / 6 / 7 / 36 / 37 / 41 / 42$ reference tag is the first group; number $2 / 3 / 7 / 8 / 37 / 38 / 42 / 43$ reference tag is the second group and so on.

Thus, we can come to each reference tag number $\mathrm{j}$ contained in the number t group:

as $\mathrm{t}^{\text {th }}$ group's reference tag, which is also used as a reference between groups. Amount s stands for the number of reference tags in this group. In our prototype, $s=8$. After calculating the group with maximal WG value, this group number is regarded as a position location of a pending positioning tag.

Pseudo-code of the algorithm:

1. for $\mathrm{i}=1$ to $\mathrm{u}$

2. $\operatorname{SumE}=0$;

3. for $\mathrm{j}=1$ to $\mathrm{n}$

4. $E_{j}=\sqrt{\sum_{i=1}^{m}\left(\theta_{i}-S_{i}\right)^{2}}$;

5. $\operatorname{Sum} E=\operatorname{Sum} E+\left(1 / E_{j}\right)^{2}$;

6. end

7. for $\mathrm{j}=1$ to $\mathrm{n}$

8. $W_{j}=\left(1 / E_{j}\right)^{2} /$ SumE;

9. end

10. for $\mathrm{t}=1$ to $\mathrm{T}$

11. $\mathrm{WG}_{\mathrm{t}}=$ sum of $\mathrm{W}$ values contained in this group;

12. end

13. Location of number i tracking tag $=$ subscript of maximal WG;

14. end

With consideration of a very few cases, two groups with relatively maximal values might have the equaled $\mathrm{WG}$ value. In this case, we chose the group with smallest amount to be the positioning location.

In a certain application scenario, the amounts of RF readers $(\mathrm{m})$ and reference tags(n) and groups(T) are constant; the amount of tracking $\operatorname{tags}(\mathrm{u})$ is variable. Thus, the time complexity of the algorithm is $O(n)$.

Note: ALLGET does not calculate coordinate of tracking tag, but locates the tracking tag in a small area. In previous case, the way of grouping is only based on the prototype system. Algorithm can be also accomplished by using other different methods of grouping to reach different area locations' demand. As soon as select the appropriate grouping method, algorithm can be easy to modify and expand according to different space division and arrangement of reference tag scenario.

\section{Experimental Results and Performance Evaluation}

We conducted a series of experiments to evaluate positioning performance of the ALLGET system in different 
situations. We adapted MATLAB to carry out a simulation experiment. In the simulation environment, we set multiple RFID reader devices and varied amounts of reference tags and used the "distance-loss" formula to establish the signal strength model of reader device.

The "distance-loss" formula is a theoretical model that reflects the relationship between the received signal strength and the geometric distance. Generally, the formula is similar as below [7]:

$$
(p)_{d B m}=-10 n \times \lg (d)+\varepsilon
$$

In this formula, $\mathrm{d}$ is the distance between the reader device and the tag; $n$ is the path loss exponent. The value of $n$ is bigger along with the signal strength decreases, distance goes longer and the loss rate becomes greater, which depends on the specific environment of dissemination. $\varepsilon$ is a normal distribution function with a mean of $\mu$ and variance of $\sigma$, which acts as a signal shielding factor. Whenever signal travels across different block objects, $\varepsilon$ changes accordingly. Taking into consideration of the actual situation, we set 0 as the mean of environmental disturbance amount $\varepsilon$. We conducted several signal samples in order to get the final result with calculating mean value.

We used random function to generate pending positioning location. Since it was impossible to get the pending positioning target completely and randomly distributed throughout the environment in the real-world scenarios, such as under the shelf, we had to limit the random function and generate anchor node that was more consistent with the actual situation.

We used accuracy rate as a reference for positioning performance. It had been set that the number of all the pending positioning tag in the system is $\mathrm{N}$ and the accuracy amount in positioning system is $\mathrm{C}$ (see below)

$$
\mathrm{R}=\frac{C}{N}
$$

Taking into consideration that a shelf could lay approximately 3000 books in maximum in the real situation, we could use 3000 as a positioning tag amount in the following experiments. In order to reduce system errors, each experiment ran randomly five times in different pending positioning tag to locate the target. Only the correct ratio of arithmetic mean was used as the final result.

In contrast, LANDMARC system's positioning output was compared under the same condition. We adjusted the number of nearest neighbor tag in LANDMARC to adapt our scene. After the experiment, the system achieved the highest accuracy when $\mathrm{k}=8$. Therefore, the LANDMARC system used $\mathrm{k}=8$ to locate as comparison in the following compared experiments.

\subsection{Effect of the Number of Readers}

The major problem of using RFID technology to locate was the uncertainty of the received signal strength. This uncertainty could be caused by the environment or it might be caused by the device itself. In addition, RFID's NLOS feature caused multipath problem, indicating that signals reach the reader device from several different paths. This made changes to the signals that were received by the reader device. More often the reader device used, the further impact of this change reduced.

Figure 3 shows the positioning accuracy rate in an ALLGET system under different reader devices' system.

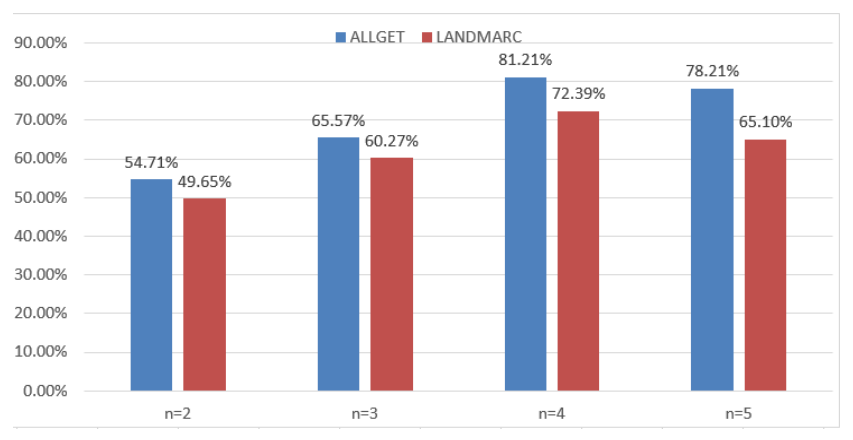

Figure 3. Accuracy rate under different number of readers.

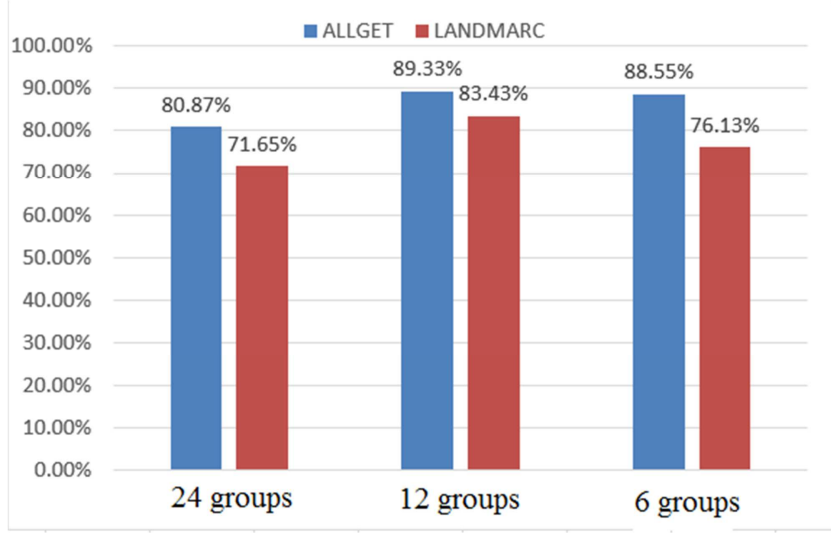

Figure 4. Accuracy rate under different density of reference tags.

As can be seen that the number of reader device increased, the system's positioning accuracy improved gradually. When the number of reader devices was four, the positioning accuracy reached the highest. In the follow-up experiment, it was found that more reader devices did not improve the system's positioning effect much, which tended to increase within a narrow range. Since reader device is relatively expensive, using more reader devices was likely to raise higher cost to the system. Therefore, it was not necessary to use so many reader devices. In the follow-up experiments, we all used four reader devices to carry out the location tasks.

\subsection{Effect of the Density of Reference Tags}

The amount of reference numbers indicates whether the reference points in the system is intensive. The more reference points, the more availability to be compared with the neighbor information in the positioning system. As for ALLGET system, intensive reference tag per unit in the space means that there are more groups and smaller regions. Thus, the location accuracy in the system is higher and more precise. Due to the change of the reference tag, the system's accuracy rate will change as well. We tried different placements of reference tags 
in the same space range.

In the same region, we tried three cellular cases, including 70 references tags divided into 24 groups, 40 reference tags divided into 12 groups and 24 reference tags divided into 6 groups. Obviously, the smaller the region an individual group has indicating the higher accuracy is in the system location.

Figure 4 shows the positioning accuracy rate of different reference tags in the system with density. It could be seen that the ALLGET system's accuracy rate was better than the accuracy in the LANDMARC system under different situations. Besides, there were two trends. On the one hand, the higher requirement on positioning system, the accuracy rate of position tended to be declined. On the other hand, reducing accuracy's requirement would reduce the amount of reference tags, and the reduction of reference tags reduces the accuracy rate of position.

\subsection{Influence of the Environmental Factors}

In the practical use, the interference between environment and signals is an important factor that affects the system performance. In different uses or different periods, the disturbances on environment are changing. In our model, the variance of the normal distribution function $\varepsilon$ represents the amount of environmental disturbance. We carried out several experiments according to different variances.

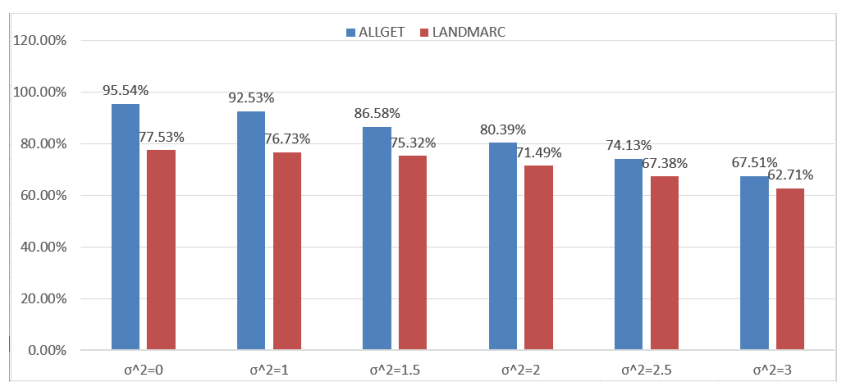

Figure 5. Accuracy rate under different $\sigma$ value.

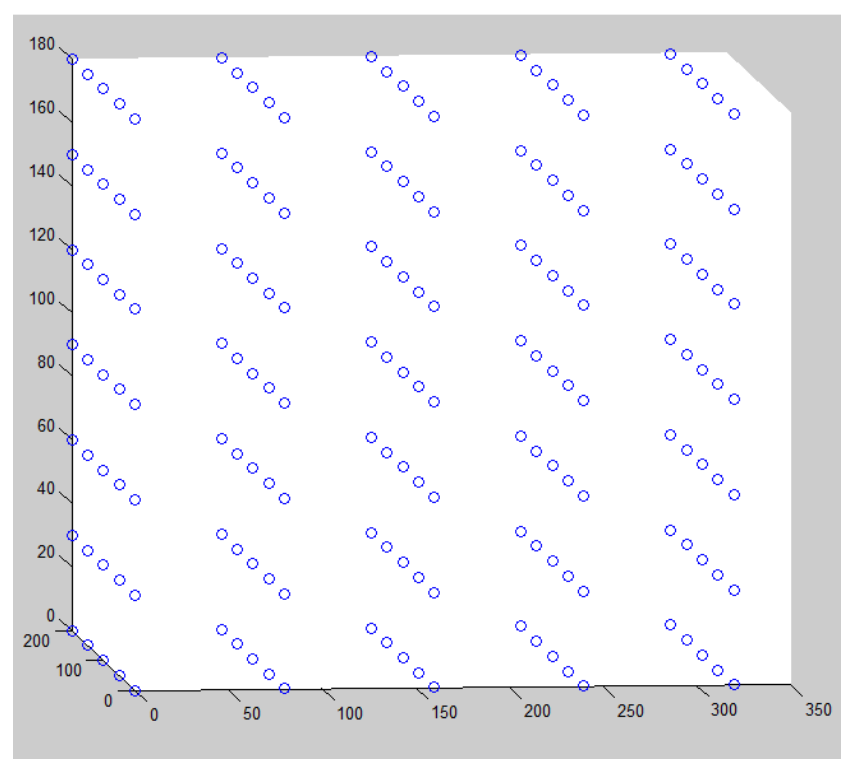

Figure 6. Schematic diagram of multifaceted reference tag array.
Figure 5 is the results from ALLGET and LANDMARC's positioning system under imitating different noise environments. We had calculated the positioning accuracy of the system from $\sigma^{2}=0$ (no noise) to $\sigma^{2}=3$ (high noise environment). Clearly, the relationship between the system's accuracy rate and the level of noise environments was negatively related. It could be seen that ALLGET system's accuracy rates on noise environments are all higher than the level of noise environments in LANDMARC system. Especially, ALLGET system performance tended to be significantly higher than the results from LANDMARC system under the low noise environment.

\section{Conclusions and Future Research}

This article introduces a region-positioning algorithm that is based on RFID technology. Additionally, it builds up an original system based on the scene of intelligent shelf. Compared with the coordinate calculation based on nearest neighbor tag of LANDMARC system and its advanced algorithm, ALLGET system's regional positioning algorithm based on the groups of reference tags gains better results with practicality. Since there is no need to record each point, the system's deployment is more convenient. On the other hand, ALLGET algorithms could achieve higher accuracy rate than LANDMARC system in different circumstances. This suggests that ALLGET algorithm's positioning system has better positioning effect and environmental adaptability.

The main advantage of grouping reference tags is to better avoid the positioning errors due to the environmental impact. Compared with the data in each group, although some signal tags in the same group may be affected, the remaining signal tags in this group are likely to balance such situations. During data comparison, the use of metrics could further reduce the error due to the uncertainty of signal strength.

There are so many factors affecting ALLGET system's positioning effect. However, generally speaking, there are two issues affecting the positioning precision rate and positioning accuracy rate of the system. Factors that affect the positioning precision of the system mainly depend on the way of grouping reference tags. This includes the size of regional grouping, shape and the amount of reference tag. Smaller group and more reference tags could improve the precision of the positioning system, which can make the pending positioning location target at much smaller areas. The major factor that affects the positioning accuracy rate is whether semaphore that reflects the actual location could be collected. In the whole system, reducing the environmental impact on signal propagation and increasing the precision of signal strength from the reader device could both improve the system's positioning accuracy rate. Therefore, using more sensitive reader devices to signal strength, choosing an appropriate shelf or placing a reference tag and pending position in a more readable way are effective approach to reduce the environmental impact. The intensive tag distribution could affect signal transmission. Thus, the scene asking for a higher precision tends to have a declining 
positioning accuracy rate. In the practical use, it is necessary to select an appropriate positioning precision according to the actual situation.

In our previous experiment, prototype system assumed the scene was on a bookshelf, which only adapted two surface of reference tag array. In fact, scenes such as libraries, warehouses can be convenient to get arranged based on the grouping reference tags as shown in the figure 6 of multifaceted reference tag array. In this case, ALLGET algorithm's positioning result is able to reflect the target location in a three-dimensional space. Moreover, grouping for the reader devices could lead to different ways of positions and effects. Currently, we are conducting relevant researches and experiments.

\section{References}

[1] Ni L M. Liu Y. Lau Y C. et al. "LANDMARC: indoor location sensing using active RFID". In Proceedings of the first IEEE International Conference on Pervasive Computing and Communications (Per Com 2003). Texas, 2003: 407-415.

[2] Khan M A, Antiwal V K. "Location Estimation Technique using Extended 3-D LANDMARC Algorithm for Passive RFID Tag". In Proceedings of IEEE Advance Computing Conference. Patiala, 2009: 249-253.

[3] V. Chandrasekhar and W. Seah. "An area localization scheme for underwater sensor networks". In Proc. Of the IEEE OCEANS Asia Pacific Conference, Singapore, 2006.
[4] P. Bahl and V. N. Padmanabhan, "RADAR: An Inbuilding RF-based User Location and Tracking System", Proceedings of IEEE INFOCOM 2000, Tel-Aviv, Israel (March 2000).

[5] Nissanka B. Priyantha, Anit Chakraborty, and Hari Balakrishnan. "The cricket location-support system". In Proceedings of MOBICOM 2000, pages32-43, Boston, MA, August 2000. ACM, ACM Press.

[6] Jeffrey Hightower, Chris Vakili, Caetano Borriello, and Roy Want, "Design and Calibration of the Spot ON AD-Hoc Location Sensing System", UWCSE 00-02-02, University of Washington, Department of Computer Science and Engineering, Seattle.

[7] Theodore S Rappaport. "Wireless Communications Principles and Practices". Second Edition Publishing House of Electronics Industry, 2004. 161.

[8] C. R. Medeiros, J. R. Costa and C. A. Fernandes. "RFID smart shelf with confined detection volume at UHF", IEEE Antennas Wireless Propag. Lett., vol. 7, pp.773 -776 2008.

[9] Jeffrey Hightower and Gaetano Borriello, “A Survey and Taxonomy of Location Sensing Systems for Ubiquitous Computing," CSE 01-08-03, University of Washington, Department of Computer Science and Engineering, Seattle, WA, Aug 2001

[10] Shuyuan Pan, Zhiying Yang. A Count Based Algorithm for Redundant Reader Elimination in RFID Application System, Intelligent System Design and Engineering Applications (ISDEA), 2013 Third International Conference on , vol., no., pp.30,33, 16-18 Jan. 2013. 